\title{
Matrix metalloproteinase-2 in the development of diabetic retinopathy and mitochondrial dysfunction
}

\author{
Ghulam Mohammad and Renu A Kowluru
}

In the pathogenesis of diabetic retinopathy, retinal mitochondria become dysfunctional resulting in accelerated apoptosis of its capillary cells. Matrix metalloproteinase-2 (MMP2) is considered critical in cell integrity and cell survival, and diabetes activates MMP2 in the retina and its capillary cells. This study aims at elucidating the mechanism by which MMP2 contributes to the development of diabetic retinopathy. Using isolated bovine retinal endothelial cells, the effect of regulation of MMP2 (by its siRNA and pharmacological inhibitor) on superoxide accumulation and mitochondrial dysfunction was evaluated. The effect of inhibiting diabetes-induced retinal superoxide accumulation on MMP2 and its regulators was investigated in diabetic mice overexpressing mitochondrial superoxide dismutase (MnSOD). Inhibition of MMP2 ameliorated glucose-induced increase in mitochondrial superoxide and membrane permeability, prevented cytochrome $c$ leakage from the mitochondria, and inhibited capillary cell apoptosis. Overexpression of MnSOD protected the retina from diabetes-induced increase in MMP2 and its membrane activator (MT1-MMP), and decrease in its tissue inhibitor (TIMP-2). These results implicate that, in diabetes, MMP2 activates apoptosis of retinal capillary cells by mitochondrial dysfunction increasing their membrane permeability. Understanding the role of MMP2 in the pathogenesis of diabetic retinopathy should help lay ground for MMP2-targeted therapy to retard the development of retinopathy in diabetic patients.

Laboratory Investigation (2010) 90, 1365-1372; doi:10.1038/labinvest.2010.89; published online 17 May 2010

KEYWORDS: diabetic retinopathy; MMP2; mitochondria dysfunction; oxidative stress

Diabetic retinopathy, a microvascular disease, causes progressive damage to the retina, and if not controlled, could lead to blindness. Although many hyperglycemia-initiated metabolic and functional abnormalities are implicated in the pathogenesis of diabetic retinopathy, the exact mechanism of its development remains elusive. Studies have shown that retinal capillary cells and other nonvascular cells undergo accelerated apoptosis before the appearance of histopathological lesions that are characteristic of diabetic retinopathy, ${ }^{1-4}$ but the mechanism responsible for their loss is not clear.

In the development of diabetic retinopathy, oxidative stress has a major role, and sustained production of mitochondrial reactive oxygen species (ROS) is considered to be the active mediators in the regulation of apoptosis. ${ }^{5-8}$ Previous studies have postulated a link between mitochondrial damage and the development of diabetic retinopathy. ${ }^{9-14}$ Regulation of mitochondrial ROS production has beneficial effects in preventing the activation of the major pathways that are implicated in the development of diabetic retinopathy, including protein kinase $C$ and polyol pathway, ${ }^{5,15,16}$ but the exact mechanism involved in the pathogenesis of diabetic retinopathy is unclear.

Matrix metalloproteinase-2 (MMP2), one of the most ubiquitous members of the MMP family, cleaves collagen type IV of the extracellular matrix maintaining equilibrium between matrix synthesis and degradation, thus providing a critical role in cell integrity and cell survival. ${ }^{17}$ Clinical and experimental studies have shown increased expression of MMP2 in diabetic subjects. ${ }^{18-22}$ Recently, we have shown a significant role of MMP2 in the development of diabetic retinopathy; our results have shown that the activation of MMP2 is under the control of superoxide. ${ }^{23}$ However, the mechanism by which MMP2 activation is controlled in the retina in diabetes needs to be evaluated.

This study is designed to test the hypothesis that MMP2 contributes to the pathology of diabetic retinopathy by inducing mitochondrial dysfunction and apoptosis of retinal

Department of Ophthalmology, Kresge Eye Institute, Wayne State University, Detroit, MI, USA

Correspondence: Dr RA Kowluru, PhD, Department of Ophthalmology, Kresge Eye Institute, Wayne State University, 4717 St Antoine, Detroit, MI 48201, USA.

E-mail: rkowluru@med.wayne.edu

Received 29 September 2009; revised 2 January 2010; accepted 29 January 2010 
capillary cells. Using both in vivo and in vitro models of diabetic retinopathy, we investigated the possible mechanism by which MMP2 could contribute to its development. Using isolated retinal endothelial cells in culture, the effect of regulation of MMP2 is determined on ROS production, mitochondrial dysfunction, and apoptosis. To confirm our in vitro results, we investigated the effect of regulation of superoxide that the retina experiences in diabetes on retinal MMP2 activity and its regulators in mice overexpressing mitochondrial superoxide dismutase $(\mathrm{MnSOD})$.

\section{MATERIALS AND METHODS Retinal Endothelial Cell Isolation and Culture}

Endothelial cells were isolated from the bovine retina and cultured on polystyrene dishes coated with $0.1 \%$ gelatin. Endothelial cells were grown in Dulbecco's modified Eagle's medium (DMEM) containing 15\% fetal bovine serum and $1 \%$ antibiotic/antimycotic at $37^{\circ} \mathrm{C}$ in $5 \% \mathrm{CO}_{2} \cdot{ }^{13,24}$ Cells from the fourth to the seventh passage were incubated in DMEM containing $2 \%$ heat-inactivated fetal calf serum, $10 \%$ Nu-serum, $50 \mu \mathrm{g} / \mathrm{ml}$ heparin, $1 \mu \mathrm{g} / \mathrm{ml}$ endothelial growth supplement, and antibiotic/antimycotic supplemented with 5 or $20 \mathrm{mM}$ glucose for 4 days in the presence or absence of $10 \mu \mathrm{M}$ of MMP2 inhibitor $\mathrm{I}^{23}$ (cis-octadecenoyl- $N$ hydroxylamide oleoyl- $N$-hydroxylamide; EMD Biosciences, Gibbstown, NJ, USA). The medium and the inhibitors were replaced every other day. At the end of incubation, the medium was collected, and the cells were washed with phosphate-buffered saline (PBS) and collected. Osmotic controls included cells incubated under identical experimental conditions with $20 \mathrm{mM}$ mannitol instead of $20 \mathrm{mM}$ glucose.

\section{Mice}

Groups of age-matched (8-10 weeks) mice overexpressing $\mathrm{MnSOD}(\mathrm{Tg})$ and their wild-type (WT) littermates were made diabetic by streptozotocin injection $(55 \mathrm{mg} / \mathrm{kg}$ BW) for 5 consecutive days. ${ }^{10,14}$ The mice, which presented blood glucose levels of $\geq 250 \mathrm{mg} / \mathrm{dl} 3$ days after the last injection were considered as diabetic. Mice were killed at $\sim 6$ months after induction of diabetes by an overdose of pentobarbital $(120 \mathrm{mg} / \mathrm{kg} \mathrm{BW})$. One eye was used to isolate the retina (under dissecting microscope) for biochemical assay, and the other eye was used for immunohistochemistry by incubating it in $10 \%$ paraformaldehyde for $30 \mathrm{~min}$. The eye was washed with PBS, fixed in OCT, and immediately frozen in liquid nitrogen for sectioning. These mice are being routinely used in our laboratory, and phenotype details are reported in our previous publications. ${ }^{14,15}$ Treatment of the animals conformed to the Association for Research in Vision and Ophthalmology Resolution on the Use of Animals in Research.

\section{Transfection of Cells with MMP2-siRNA}

Endothelial cells (60-80\% confluent) from the third to the fourth passage were transfected using transfection reagent kit and MMP2 siRNA (Santa Cruz Biotechnology, Santa Cruz, CA, USA) as routinely carried out in our laboratory. ${ }^{25}$ The transfection complex, prepared by adding MMP2 siRNA and siRNA transfection reagent, was incubated for $30 \mathrm{~min}$ at room temperature, and the cells were incubated with this transfection complex for $8 \mathrm{~h}$ at $37^{\circ} \mathrm{C}$. Parallel incubations were carried out using nontargeting scrambled siRNA. After the transfection, cells were rinsed with PBS and incubated in 5 or $20 \mathrm{mM}$ glucose media for 4 days.

\section{Isolation of the Mitochondria and Cytosol}

Mitochondria and cytosol fractions were prepared from retinal endothelial cells by differential centrifugation method as previously used by us. ${ }^{10}$ Protein was determined by the Bicinchoninic Acid protein assay (Sigma-Aldrich, St Louis, MO, USA).

\section{Gelatin Zymography}

Gelatenolytic activity of MMP2 was estimated in culture media or in the retina by zymography technique. ${ }^{23}$ The samples (10-20 $\mu \mathrm{g}$ protein) were electrophoresed under nonreducing conditions onto $10 \%$ SDS-PAGE gels polymerized with $1 \mathrm{mg} / \mathrm{ml}$ gelatin. After washing the gel with $2.5 \%$ Triton X-100, it was incubated overnight at $37^{\circ} \mathrm{C}$ in substrate buffer containing $50 \mathrm{mM}$ Tris- $\mathrm{HCl}, \mathrm{pH} 8.0,5 \mathrm{mM}$ $\mathrm{CaCl}_{2}$, and $0.02 \% \mathrm{NaN}_{3}$. The gel was stained with Coomassie blue stain (Simply Blue Safe Stain; Invitrogen, Carlsbad, CA, USA), and this was followed by destaining with distilled water. The band intensity of the active MMP2 $(67 \mathrm{kDa})$ band was quantified using Un-Scan-It Gel digitizing software.

\section{Western Blot Analysis}

Protein $(25-40 \mu \mathrm{g})$ was subjected to SDS-PAGE and transferred to nitrocellulose membrane. Immunodetection was performed using antibodies against cytochrome $c$ and poly-ADP ribose polymerase, PARP1/2 (Santa Cruz Biotechnology). Cox IV and $\beta$-actin were used as the loading controls for mitochondria and cytosol fractions, respectively.

\section{Gene Expression}

Gene expression was determined using conventional semiquantitative PCR as routinely performed in our laboratory. ${ }^{23,25} \mathrm{cDNA}$ template $(0.5 \mu \mathrm{g})$ was added to $10 \mathrm{pmol}$ of forward and reverse primers (designed using Applied Biosystems software Primer Express 3.0 and synthesized by Integrated DNA Technologies, Coralville, IA, USA), and 1 Unit of GoTaq DNA polymerase (Promega, Madison, WI, USA). The standard PCR conditions included $2 \mathrm{~min}$ at $50^{\circ} \mathrm{C}$ and $10 \mathrm{~min}$ at $95^{\circ} \mathrm{C}$, followed by 35 cycles of denaturation at $95^{\circ} \mathrm{C}$ for $15 \mathrm{~s}$, and $1 \mathrm{~min}$ of annealing at $60^{\circ} \mathrm{C}$. The primers sequences for bovine cells were: forward $5^{\prime}$-CCTCCTGCTGG GGACGCTGC- $3^{\prime}$ and reverse $5^{\prime}$-AGTCCTGGTGGCCTGAC 
GGG-3' for TIMP2; and forward 5'-AACATCAAAGTCTG GGAAGG- $3^{\prime}$ and reverse $5^{\prime}$-GAAGTTCTCGGTGTCCATC CA- $3^{\prime}$ for $M T 1-M M P$. The primers used for mouse retina were: forward $5^{\prime}$-AGAGACTGGCTTAGGAGGGC- $3^{\prime}$ and reverse $5^{\prime}$-ATGTCAGACAACCCGAGTCC- $3^{\prime}$ for $M M P 2$; forward $5^{\prime}$-AGTCAGGGTCACCCACAAAG- $3^{\prime}$ and reverse $5^{\prime}$-GCATTGGGTATCCATCCATC- $3^{\prime}$ for $M T 1-M M P$; and forward $5^{\prime}$-CTTCTCCGCCGGGTGCACTG- $3^{\prime}$ and reverse $5^{\prime}$-CAGCAGCGTGGCTAGCAGCA-3' for T1MP-2. After the amplification, the DNA was run on a $1.2 \%$ agarose gel, and a $100 \mathrm{bp}$ DNA ladder was simultaneously run on each gel. The bands were visualized using the UVP Bio-Doc it Imaging System (UVP LLC, Upland, CA, USA), and band intensities were quantified by Un-Scan-It software.

\section{Mitochondrial ROS Generation}

MitoTracker Red $\mathrm{CM}-\mathrm{H}_{2}$ XROS (Molecular Probes; Invitrogen), a cell-permeable dye that sequesters in the mitochondria and emits fluorescence when oxidized was used to measure mitochondrial ROS. After the desired incubations, the cells were washed with fresh medium to remove nonadherent cells, and incubated with $400 \mathrm{nM}$ MitoTracker Red for $30 \mathrm{~min}$. This was followed by extensive washing with DMEM, and mounting in Vectastain-DAPI mounting media (Vector Laboratories, Burlington, CA, USA). The fluorescence was visualized using Olympus BX50 fluorescent microscope.

\section{Mitochondrial-Permeability Pore Transition}

Mitochondrial membrane potential collapses by a sudden increase in the permeability of the inner membrane to small ions and molecules resulting in swelling. This collapse of mitochondrial membrane potential was quantified spectrophotometrically using 5-20 $\mu \mathrm{g}$ protein mitochondrial protein as previously reported by us. ${ }^{10}$ The transition induced by $400 \mu \mathrm{M}$ calcium chloride containing $75 \mu \mathrm{M}$ $t$-butylhydroperoxide was measured at $540 \mathrm{~nm}$. The values were expressed as a percentage of swelling with respect to the maximum swelling achieved by exposure to external calcium.

\section{Mitochondrial Membrane Potential}

To measure mitochondrial membrane potential, cationic dye JC-1 (MitoPT, Immunohistochemistry Technologies, Bloomington, MN, USA) was used. At the end of the incubation period, medium was removed, the cells were rinsed with DMEM and incubated with $1 \times$ MitoPT staining for $15 \mathrm{~min}$ at $37^{\circ} \mathrm{C}$. The cells were washed and visualized under Olympus BX50 fluorescent microscope.

\section{Apoptosis}

Apoptosis was determined by Cell Death Detection ELISA $^{\text {PLUS }}$ (Roche Diagnostics, Indianapolis, IN, USA) using antibodies directed against DNA and histones, respectively, as routinely used in our laboratory. ${ }^{13,23}$

\section{Immunohistochemical Analysis}

Cryosections $(10 \mu \mathrm{m})$ prepared from the mouse retina were blocked by incubating them with the blocking reagent containing 2.5\% bovine serum albumin and 5\% serum from the host animal of the secondary antibody in PBS, for $30 \mathrm{~min}$ in a moist chamber. The slides were rinsed with PBS and incubated for $1 \mathrm{~h}$ with anti-MMP2 (goat polyclonal, Santa Cruz Biotechnology). After rinsing the slides with PBS, they were incubated with the secondary antibody (anti goat-FITC conjugated) for $1 \mathrm{~h}$. The slides were then rinsed with $50 \mathrm{mM}$ Tris-HCl buffer ( $\mathrm{pH}$ 7.5), and counterstained with DAPI (Vector Laboratories). The sections were imaged using the Olympus BX50 fluorescent microscope $(\times 10$ magnification). Control slides included staining of the samples that were incubated under identical conditions, except the primary antibody.

\section{Statistical Analysis}

The results are presented as mean \pm s.d. and analyzed statistically using the nonparametric Kruskal-Wallis test, followed by the Mann-Whitney test for multiple group comparisons. Similar conclusions were achieved using ANOVA with Fisher's or Tukey's test.

\section{RESULTS}

\section{Retinal Endothelial Cells in Culture}

High glucose exposure activates MMP2 and damages the mitochondria

Incubation of retinal endothelial cells in high glucose medium, as expected, increased MMP2 gelatinase activity by about $30-40 \%$ compared with cells incubated in normal glucose medium (Figure 1a). In the same cell preparations, the gene expression of its membrane activator, MT1-MMP, was increased by $25 \%$ and that of TIMP-2 (intracellular inhibitor) was decreased by 70\% (Figure 1b). ROS levels were significantly increased as evidenced by enhanced staining of MitoTracker Red (Figure 2). Mitochondrial swelling was increased by more than four fold (Figure 3a), and its membrane-permeability transition, as determined by cationic fluorescent dye, was also increased significantly in cells incubated in high glucose compared with cells incubated in normal glucose (Figure 3b). The leakage of cytochrome $c$ from the mitochondria into the cytosol was increased by $40 \%$, and this was accompanied by a significant decrease in cytochrome $c$ expression in the mitochondria (Figure 4). Apoptosis was increased by more than fourfold (Figure 5a), and the cleavage of PARP into its $85 \mathrm{kDa}$ subunit by $40 \%$ (Figure 5b).

\section{Inhibition of MMP2 prevents glucose-induced mitochondrial dysfunction}

Transfection of endothelial cells with MMP2-siRNA prevented glucose-induced increases in MMP2 activity and MT1-MMP gene expression, and decrease in TIMP-2; however, transfection of cells with scrambled siRNA had no effect on any of these 
a
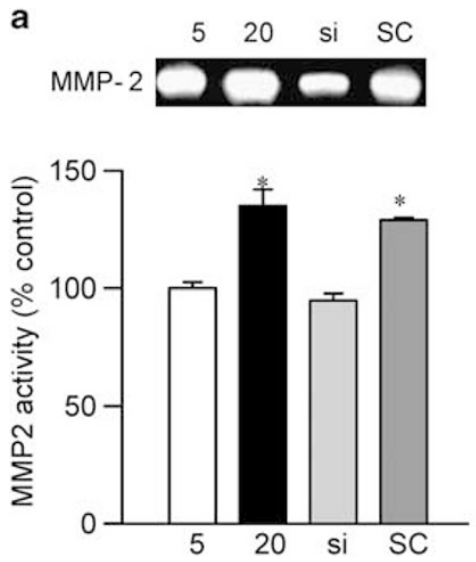

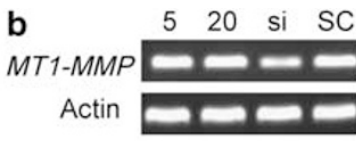

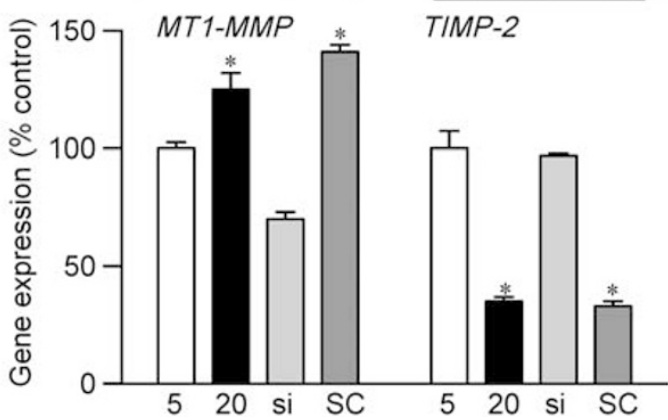

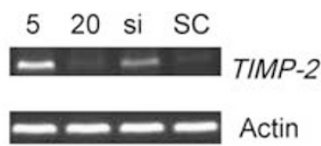

TIMP-2

Figure 1 Effects of genetic manipulation of MMP2 on its glucose-induced activity and the regulators in retinal endothelial cells: bovine retinal endothelial cells from the fourth passage were incubated in 5 or $20 \mathrm{mM}$ glucose medium for 4 days in the presence or absence of MMP2-siRNA. At the end of incubation, the medium was collected for gelatinolytic activity, and RNA was isolated from the cells by TRizol. (a) The gelatinase activity of MMP2 was determined in the medium by zymography technique. (b) Gene expressions of MT1-MMP and TIMP-2 were quantified by semi-quantitative PCR using the primers given in the 'Materials and methods' section and were adjusted to the mRNA levels of $\beta$-actin in each sample. Each measurement was performed in at least three different cell preparations. The values, represented as mean \pm s.d., obtained from the cells incubated in $5 \mathrm{mM}$ glucose are considered as $100 \%$ (control). ${ }^{*} P<0.05$ compared with the values obtained from cells incubated in $5 \mathrm{mM}$ glucose. Both 5 and $20 \mathrm{mM}=$ cells incubated in 5 or $2 \mathrm{mM}$ glucose, respectively, si and SC=cells transfected with MMP2-siRNA or scrambled RNA, respectively, followed by incubation in $20 \mathrm{mM}$ glucose for 4 days.
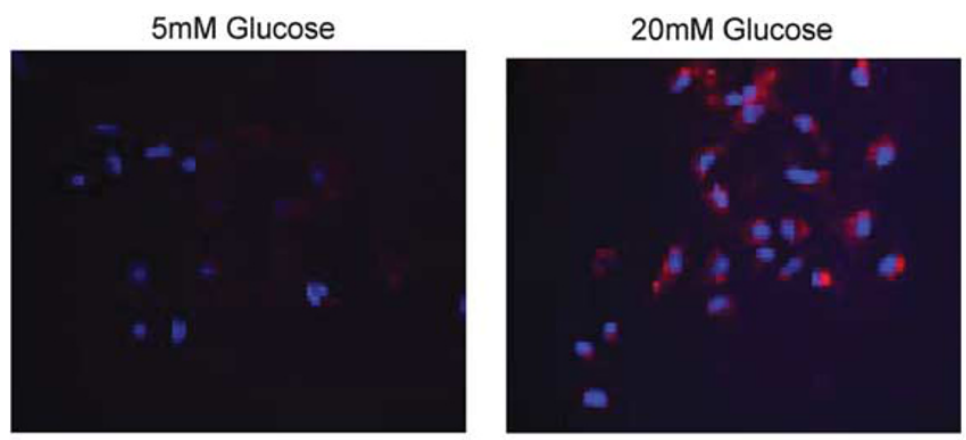

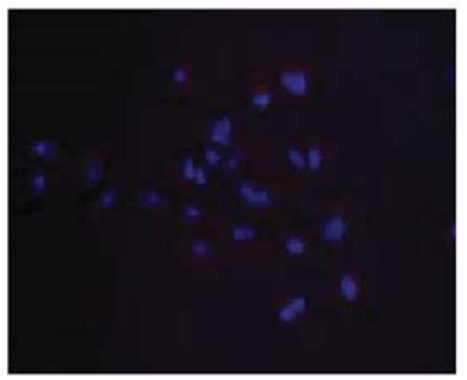

20+siRNA

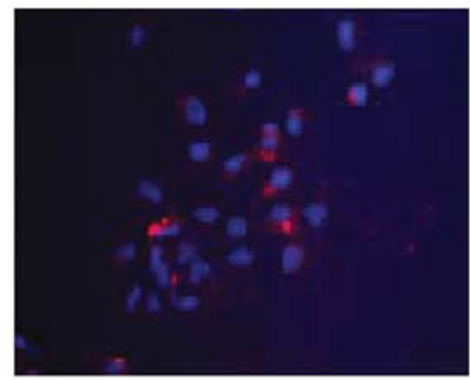

$20+$ scrambled

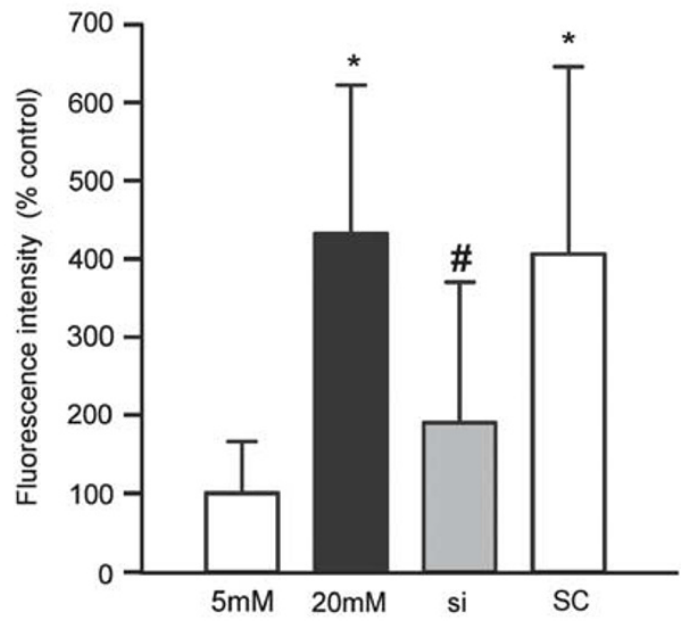

Figure 2 Effect of MMP2-siRNA on mitochondrial superoxide: retinal endothelial cells, either transfected with MMP2-siRNA or untransfected were incubated in 5 or $20 \mathrm{mM}$ glucose for 4 days. After washing the cells with phosphate-buffered saline, they were stained with $400 \mathrm{nM}$ MitoTracker Red CM-H2XROS for $30 \mathrm{~min}$. After removing the excessive stain by washing with phosphate-buffered saline, they were mounted in vectashield mounting media and observed under fluorescence microscope at $\times 20$ magnification. The red fluorescence intensity was quantified using Image $\mathrm{J}$ software (NIH) Java (TM) Platform

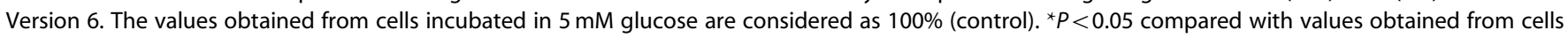
incubated in $5 \mathrm{mM}$ glucose and ${ }^{\#} P<0.05$ compared with $20 \mathrm{mM}$ glucose: 5 and $20 \mathrm{mM}=$ cells incubated in 5 or $20 \mathrm{mM}$ glucose, respectively, si and SC $=$ cells transfected with MMP2-siRNA or scrambled RNA, respectively, followed by incubation in 20 mM glucose for 4 days.

parameters (Figure 1). Glucose-induced increase in mitochondrial superoxide levels, and membrane swelling were ameliorated in cells transfected with MMP2-siRNA (Figures 2 and 3). MMP2 inhibitor, which we have shown to inhibit glucose-induced increase in MMP2 activity and apoptosis of retinal capillary cells, ${ }^{23}$ also abrogated the alterations in 
a

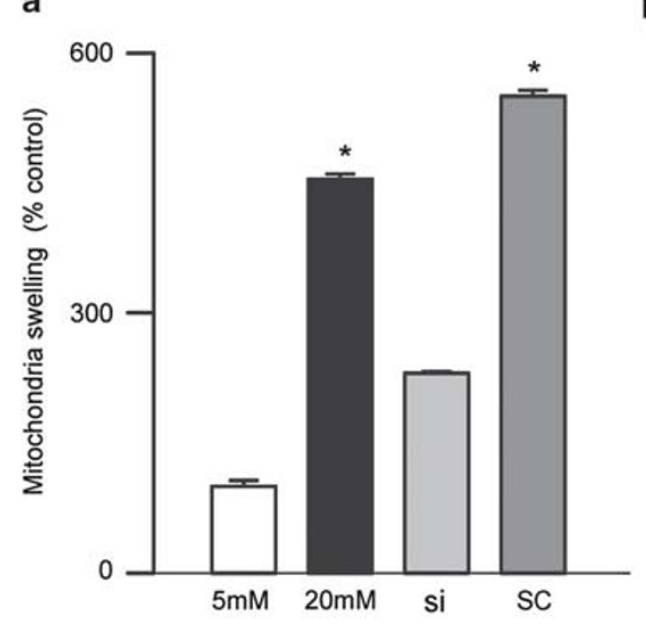

b

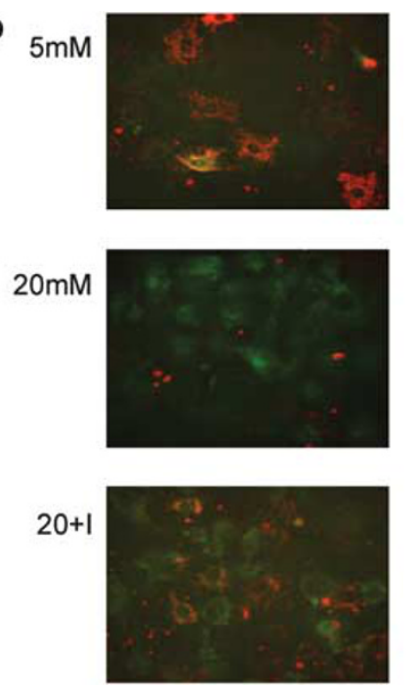

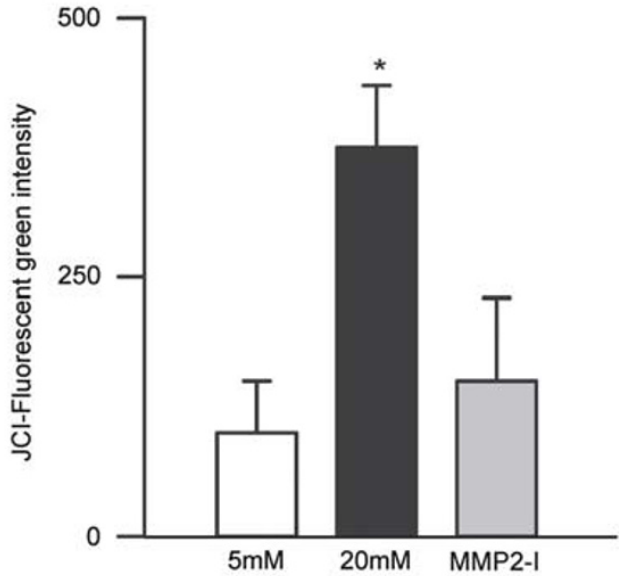

Figure 3 Effect of MMP2 inhibition on mitochondrial membranes. (a) Freshly isolated mitochondria prepared from MMP2-siRNA-transfected or MMP2siRNA-untransfected cells incubated in 5 or $20 \mathrm{mM}$ glucose for 4 days were used to determine the collapse of their membrane potential by measuring the decrease in absorbance at $540 \mathrm{~nm}$ induced by calcium chloride. The extent of swelling was calculated as a percentage of swelling with respect to the maximum swelling achieved by exposure to external calcium. (b) Mitochondrial membrane potential was determined by cationic dye JC-1 in cells incubated in 5 or $20 \mathrm{mM}$ glucose in the presence or absence of MMP2 inhibitor (MMP2-I). Green fluorescence represents the depolarized (monomer) mitochondria and red fluorescence represents the hyperpolarized (J aggregates) mitochondria. Green fluorescence intensity was determined using the Image $J$ software. Results are expressed as mean \pm s.d., ${ }^{*} P<0.05$ compared with the values obtained from cells incubated in $5 \mathrm{mM}$ glucose. Both 5 and $20 \mathrm{mM}=$ cells incubated in 5 or $20 \mathrm{mM}$ glucose, respectively, si and SC = cells transfected with MMP2-siRNA or scrambled RNA, respectively, followed by incubation in $20 \mathrm{mM}$ glucose for 4 days, and MMP2-I=cells incubated in $20 \mathrm{mM}$ glucose medium containing MMP2 inhibitor.

Cytosol

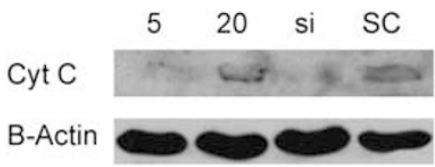

\section{Mitochondria}

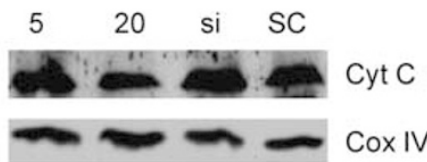

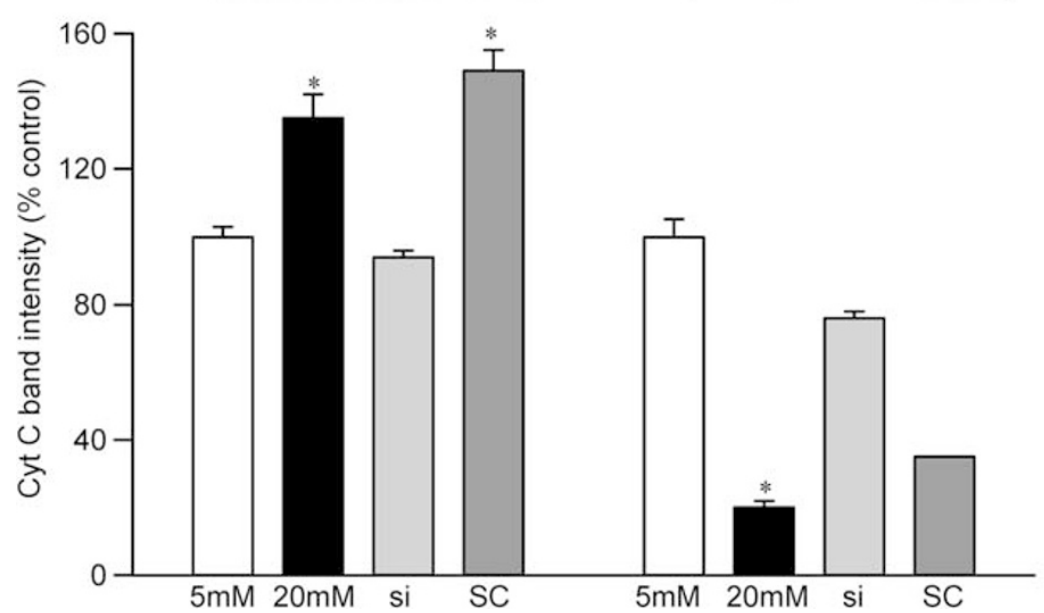

Figure 4 Effect of MMP2-siRNA on the leakage of cytochrome $c$ from the mitochondria: The mitochondrial and cytosolic fractions were prepared from freshly harvested cells by differential centrifugation. Cytochrome $c$ contents were determined by western blot in both mitochondrial and cytosolic fractions. The band intensities of cytochrome $c$ were adjusted to the expression of the $\beta$-actin or Cox IV (cytosolic and mitochondrial fractions, respectively). The western blots are representative of three different experiments. The values obtained from cells incubated in $5 \mathrm{mM}$ glucose are considered as $100 \%$. ${ }^{\star} P<0.05$ compared with cells incubated in $5 \mathrm{mM}$ glucose.

mitochondrial membrane potential (Figure 3b). Cytochrome $c$ leakage from the mitochondria into the cytosol, which is increased in endothelial cells in diabetes, ${ }^{11}$ was significantly decreased by MMP2-siRNA (Figure 4), and in the same cell preparations, glucose-induced apoptosis was also ameliorated, and the cleavage of PARP was prevented (Figure 5). 
a

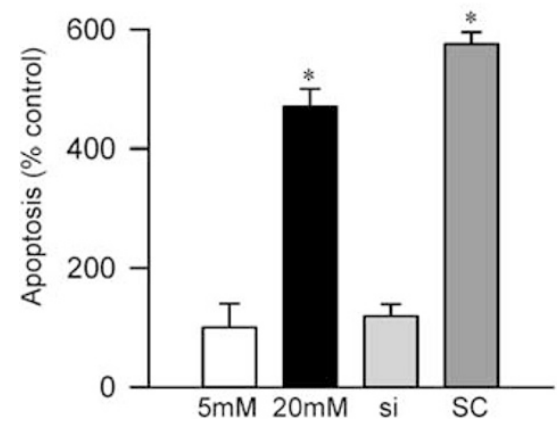

b
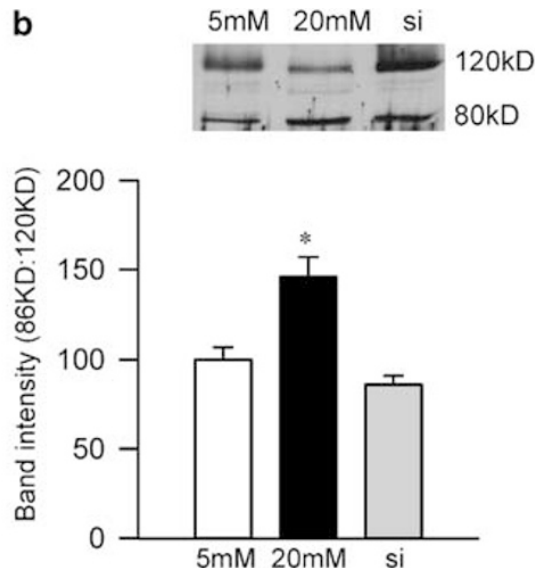

Figure 5 Effect of MMP2 inhibition on apoptosis of bovine retinal endothelial cells. (a) Apoptosis was measured by performing cell death ELISA by measuring cytoplasmic histone-associated DNA fragments using an assay kit from Roche Diagnostics. (b) PARP activation was measured by western blot technique, and the ratio of active PARP and pro-PARP was calculated. Each experiment was repeated using three separate cell preparation and the values obtained from cells incubated in $5 \mathrm{mM}$ glucose are considered as $100 \%$ (control). ${ }^{*} P<0.05$ compared with the values obtained from cells incubated in $5 \mathrm{mM}$ glucose. Both 5 and $20 \mathrm{mM}=$ cells incubated in 5 or $20 \mathrm{mM}$ glucose, respectively, si and SC=cells transfected with MMP2-siRNA or scrambled RNA, respectively, followed by incubation in $20 \mathrm{mM}$ glucose for 4 days.

a

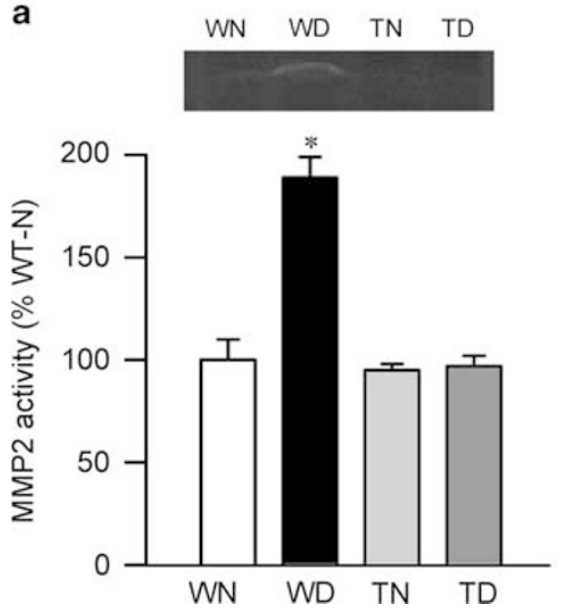

b

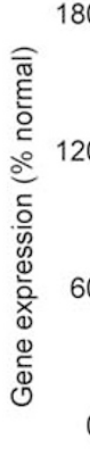

$M M P 2$
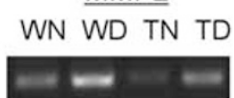

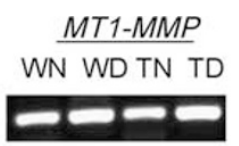

*
TIMP-2 WN WD TN TD

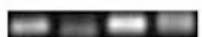

Figure 6 Effect of MnSOD overexpression on retinal MMP2 and its regulators. (a) The gelatinase activity of MMP2 was quantified by in situ zymography using 10-15 $\mu \mathrm{g}$ of retinal homogenate. (b) The gene expressions of MMP2, MT1-MMP, and TIMP-2 were quantified in the retina obtained from wild-type and MnSOD overexpressing mice maintained diabetic for 6 months and their age-matched controls. The levels of mRNA were adjusted to the mRNA levels of $\beta$-actin in each sample. Each measurement was performed at least three times. The values obtained from WT nondiabetic mice are considered as $100 \%$. Results are expressed as mean \pm s.d. of at least six mice in each group. ${ }^{\star} P<0.05$ compared with WT nondiabetic mice. WN and WD $=$ wild-type nondiabetic and diabetic, respectively, and TN and TD $=M n S O D$ transgenic mice nondiabetic and diabetic, respectively.

\section{Diabetic Mice}

Overexpression of MnSOD prevents diabetes-induced activation of MMP2 and its regulators

Diabetes in WT mice, as observed in rats, ${ }^{23}$ increased MMP2 gelatinase activity by $>80 \%$, and its gene expression was elevated by $55-60 \%$ compared with the values obtained from nondiabetic WT mice. In the same retina samples, MT1-MMP expression was increased by $60 \%$, and TIMP-2 expression was decreased by $\sim 25 \%$ (Figure 6 ). Immunostaining of MMP2, as detected by green FITC fluorescent staining, was also significantly increased in the retinal vessels and in several areas surrounding the retinal vessels in the sections obtained from WT diabetic mice compared with
WT nondiabetic mice (Figure 7). Overexpression of MnSOD prevented diabetes-induced activation of MMP2 and its regulators; MMP2 gelatinase activity and gene expressions of MMP2, MT1-MMP, and TIMP-2 were not statistically different in the retina from diabetic $\mathrm{Tg}$ and nondiabetic $\mathrm{Tg}$ mice, and the values were similar to those obtained from WT nondiabetic mice (Figure 6). The intensity of FITC staining in the sections obtained from $\mathrm{Tg}$ diabetic mice was significantly lower compared with WT diabetic mice (Figure 7).

\section{DISCUSSION}

Diabetic retinopathy remains the major cause of blindness among young adults; despite extensive research in the field, 
a

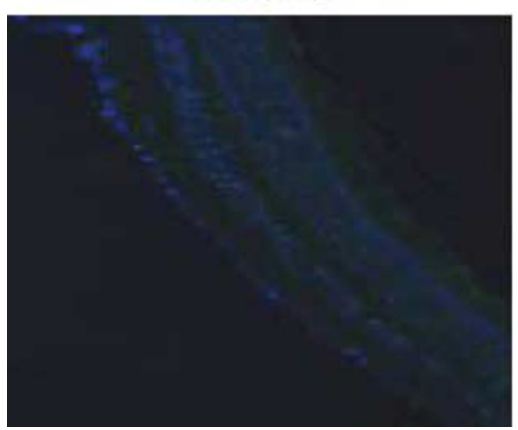

b

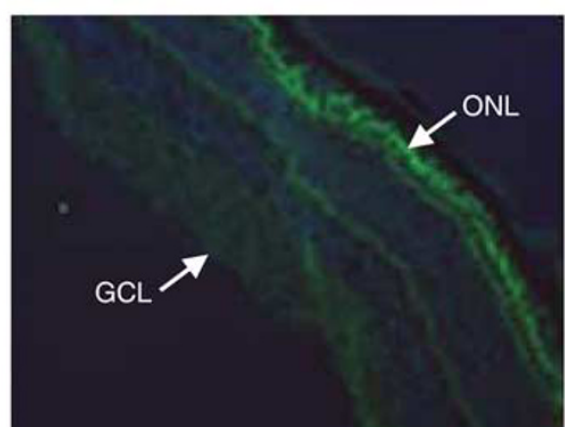

c

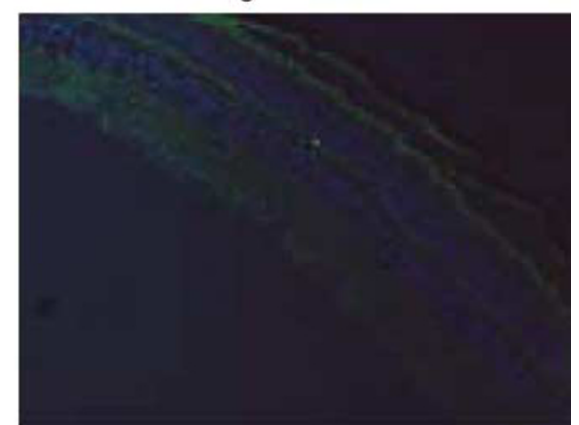

Figure 7 Immunostaining of MMP2. Cryosections from (a) WT nondiabetic (b) WT diabetic, and (c) Tg diabetic mice were subjected to immunostaining using MMP2 antibodies (green) and DAPI (blue) was used to stain the nuclei. The sections were imaged at $\times 10$ magnification using Olympus BX50 fluorescent microscope, and the image is representative of 3 or more mice in each group. GCL, ganglion cell layer; ONL, outer nuclear layer.

the precise mechanism(s) of its development remains unclear. In the development of diabetic retinopathy, accelerated apoptosis of retinal microvascular cells (pericytes and endothelial cells) and other nonvascular cells is observed before other histopathology is detectable, ${ }^{1-4}$ or loss of vision is evident, and apoptosis of capillary cells predicts the pathology associated with diabetic retinopathy. ${ }^{3}$ Our recent study has suggested a proapoptotic role for MMP2 in the loss of retinal capillary cells in diabetes, and has shown that the increase in MMP2 activity and its gene expression in diabetic rat retina and its microvessels is inhibited by therapies that inhibit capillary cell apoptosis and the development of retinopathy. $^{23}$ In this study, we elucidate the possible mechanism by which MMP2 increases apoptosis of retinal capillary cells. The exciting data demonstrate the importance of MMP2 in high glucose-mediated mitochondrial dysfunction and apoptosis; inhibition of MMP2 ameliorates glucoseinduced mitochondrial superoxide and dysfunction, and protects the mitochondria from leaking cytochrome $c$ into the cytosol resulting in the inhibition of capillary cell apoptosis. From the mouse model of diabetic retinopathy, we show that regulation of mitochondrial superoxide by overexpression of $M n S O D$, which prevents mitochondrial dysfunction and the development of diabetic retinopathy, ${ }^{10}$ also prevents diabetes-induced dysregulation of MMP2 and its regulators in the retina. Thus, dysfunction of the mitochondria could be one of the possible mechanism by which MMP2 contributes to the development of diabetic retinopathy.

Diabetes increases ROS levels in the retina and its capillary cells, and increased oxidative stress is implicated in the development of diabetic retinopathy. ${ }^{9,10,14}$ ROS, by opening the mitochondrial-permeability transition pores, alter the mitochondrial membrane potential, and this allows cytochrome $c$ to leak from the mitochondria, subsequently activating apoptosis machinery. ${ }^{26,27}$ Mitochondrial dysfunction is postulated to have a major role in the pathogenesis of diabetic retinopathy. We have shown that mitochondrial superoxide levels are elevated in the retina in diabetes, mitochondrial macromolecules are damaged, and cyto- chrome $c$ is released out of the mitochondria. ${ }^{10,11}$ Others have shown ROS to activate MMP2 in myocytes, ${ }^{28}$ and overexpression of MMP2 to increase the abnormalities in mitochondrial ultrastructure and lipid peroxidation in cardiac tissue. ${ }^{29}$ A recent study has shown a proapoptotic role for MMP2 in the loss of retinal capillary cells in diabetes; ${ }^{23}$ our data now provide a possible mechanism by which MMP2 accelerates the apoptosis of retinal capillary cells: manipulation of the MMP2 gene by its siRNA protects retinal endothelial cells from high glucose-induced increased superoxide accumulation and mitochondrial damage, and this prevents cytochrome $c$ to leak into the cytosol inhibiting the apoptosis of the cells. These results strongly suggest that MMP2 upregulation in the retina in diabetes increases apoptosis of retinal capillary cells by collapsing mitochondrial membranes. In support, MT1-MMP is reported to sensitize endothelial cells to apoptosis by caspase-3 activation, which can be abrogated by Bcl-2, thus suggesting the involvement of a mitochondrial pathway. ${ }^{30}$ Furthermore, we show that glucose-induced PARP activation in retinal endothelial cells is also under the control of MMP2. In support, the activation of MMP2 is reported to cleave the nuclear PARP, $^{31}$ and because of significant crosstalk between the nucleus and mitochondria, cleavage of PARP can result in apoptosis through the mitochondrial pathway by releasing apoptosis-inducing factor from the mitochondria, which ultimately facilitates the release of cytochrome $c .^{26,32}$

In mice overexpressing $M n S O D$, diabetes fails to increase mitochondrial damage and protects the retinal vasculature from the pathology that is characteristic of diabetic retinopathy. ${ }^{10,33}$ Our in vivo results show that protection of mitochondrial superoxide accumulation in the retina in diabetes by overexpression of $M n S O D$ also prevents elevation in MMP2 activity and its regulators, and are consistent with the in vitro results demonstrating the role of retinal mitochondrial damage in the regulation of MMP2. In support, published reports from other laboratories have implicated the role of oxidative stress in the activation of MMP2. ${ }^{34,35}$ Thus, it is conceivable to hypothesize that the 
protection of retinal mitochondrial damage in diabetes helps MMP2 from being activated, and this further keeps the apoptosis machinery in check. However, we recognize that there could be other members of the MMP family involved in the development of diabetic retinopathy, but our focus was to determine the mechanism by which MMP2, the most ubiquitous member, results in the apoptosis of retinal capillary cells in diabetes.

In summary, in this study, we present a probable mechanism that allows MMP2 to act as a proapoptotic in diabetic milieu, thus accelerating the loss of capillary cells resulting in the development of diabetic retinopathy. Understanding the role of MMP2 in the pathogenesis of diabetic retinopathy should help lay ground for MMP2-targeted therapy to retard the development of retinopathy, the sight threatening complication that diabetic patients are faced with.

\section{ACKNOWLEDGEMENTS}

We thank Mamta Kanwar and Yakov Shamailov for technical help, and Dr Sally Madsen-Bouterse for help with the transfection experiments. This study was supported in part by grants from the National Institutes of Health (NIH-EY014370), the Juvenile Diabetes Research Foundation, the Thomas Foundation, and Research to Prevent Blindness.

\section{DISCLOSURE/CONFLICT OF INTEREST}

The authors declare no conflict of interest.

1. Mizutani $M$, Kern TS, Lorenzi M. Accelerated death of retinal microvascular cells in human and experimental diabetic retinopathy. J Clin Invest 1996:97:2883-2890.

2. Kern TS, Tang J, Mizutani M, et al. Response of capillary cell death to aminoguanidine predicts the development of retinopathy: comparison of diabetes and galactosemia. Invest Ophthalmol Vis Sci 2000;41: 3972-3978.

3. Barber AJ, Lieth E, Khin SA, et al. Neural apoptosis in the retina during experimental and human diabetes. Early onset and effect of insulin. J Clin Invest 1998;102:783-791.

4. Behl $Y$, Krothapalli $P$, Desta $T$, et al. Diabetes-enhanced tumor necrosis factor-alpha production promotes apoptosis and the loss of retinal microvascular cells in type 1 and type 2 models of diabetic retinopathy. Am J Pathol 2008;172:1411-1418.

5. Brownlee $M$. The pathobiology of diabetic complications: a unifying mechanism. Diabetes 2005;54:1615-1625.

6. Hammes HP. Pathophysiological mechanisms of diabetic angiopathy. J Diabetes Complicat 2003:17:16-19.

7. Kowluru RA, Kern TS, Engerman RL, et al. Abnormalities of retinal metabolism in diabetes or experimental galactosemia. III. Effects of antioxidants. Diabetes 1996;45:1233-1237.

8. Kowluru RA, Tang J, Kern TS. Abnormalities of retinal metabolism in diabetes and experimental galactosemia. VII. Effect of long-term administration of antioxidants on the development of retinopathy. Diabetes 2001;50:1938-1942.

9. Brownlee M. Biochemistry and molecular cell biology of diabetic complications. Nature 2001;414:813-820.

10. Kanwar M, Chan PS, Kern TS, et al. Oxidative damage in the retinal mitochondria of diabetic mice: possible protection by superoxide dismutase. Invest Ophthalmol Vis Sci 2007:48:3805-3811.

11. Kowluru RA, Abbas SN. Diabetes-induced mitochondrial dysfunction in the retina. Inves Ophthalmol Vis Sci 2003:44:5327-5334.

12. Kowluru RA. Diabetic retinopathy: mitochondrial dysfunction and retinal capillary cell death. Antioxid Redox Signal 2005;7:1581-1587.
13. Kowluru RA, Atasi L, Ho YS. Role of mitochondrial superoxide dismutase in the development of diabetic retinopathy. Invest Ophthalmol Vis Sci 2006;47:1594-1599.

14. Kowluru RA, Kowluru V, Ho YS, et al. Overexpression of mitochondrial superoxide dismutase in mice protects the retina from diabetesinduced oxidative stress. Free Rad Biol Med 2006;41:1191-1196.

15. Kanwar M, Kowluru RA. Role of glyceraldehyde 3-phosphate dehydrogenase in the development and progression of diabetic retinopathy. Diabetes 2009;58:227-234.

16. Nishikawa T, Kukidome D, Sonoda K, et al. Impact of mitochondrial ROS production on diabetic vascular complications. Diabetes Res Clin Pract 2007;77:S41-S45.

17. Malemud CJ. Matrix metalloproteinases (MMPs) in health and disease: an overview. Front Biosci 2006;11:1696-1701.

18. Das A, McGuire PG, Eriqat $C$, et al. Human diabetic neovascular membranes contain high levels of urokinase and metalloproteinase enzymes. Invest Ophthalmol Vis Sci 1999;40:809-813.

19. Giebel SJ, Menicucci G, McGuire PG, et al. Matrix metalloproteinases in early diabetic retinopathy and their role in alteration of the bloodretinal barrier. Lab Invest 2005;85:567-607.

20. Jin $M$, Kashiwagi $K$, lizuka $Y$, et al. Matrix metalloproteinases in human diabetic and nondiabetic vitreous. Retina 2001;21:28-33.

21. Shiau MY, Tsai ST, Tsai KJ, et al. Increased circulatory MMP-2 and MMP-9 levels and activities in patients with type 1 diabetes mellitus. Mt Sinai J Med 2006;73:1024-1028.

22. Yang $\mathrm{R}$, Liu H, Williams $\mathrm{l}$, et al. Matrix metalloproteinase-2 expression and apoptogenic activity in retinal pericytes: implications in diabetic retinopathy. Ann NY Acad Sci 2007;1103:196-201.

23. Kowluru RA, Kanwar M. Oxidative stress and the development of diabetic retinopathy: contributory role of matrix metalloproteinase-2. Free Rad Biol Med 2009:46:1677-1685.

24. Kowluru V, Kowluru RA. Increased oxidative stress in diabetes regulates activation of a small molecular weight G-protein, H-Ras, in the retina. Mol Vis 2007;13:602-610.

25. Kowluru RA, Kanwar M. Translocation of H-Ras and its implications in the development of diabetic retinopathy. Biochem Biophys Res Commun 2009;387:461-466.

26. Kuo CT, Hsu MJ, Chen BC, et al. Denbinobin induces apoptosis in human lung adenocarcinoma cells via Akt inactivation, Bad activation, and mitochondrial dysfunction. Toxicol Lett 2008;177:48-58.

27. Phaneuf $S$, Leeuwenburgh $C$. Cytochrome $c$ release from mitochondria in the aging heart: a possible mechanism for apoptosis with age. Am J Physiol Regul Integr Comp Physiol 2002;282:R423-R430.

28. Schulz R. Intracellular targets of matrix metalloproteinase-2 in cardiac disease: rationale and therapeutic approaches. Ann Rev Pharmac Toxicol 2007:47:211-242.

29. Zhoua H-Z, Maa X, Graye MO, et al. Transgenic MMP-2 expression induces latent cardiac mitochondrial dysfunction. Biochem Biophys Res Comm 2007;358:189-195.

30. Langlois $\mathrm{S}$, Tomasso $\mathrm{G}$, Boivin $\mathrm{D}$, et al. Membrane type 1-matrix metalloproteinase induces endothelial cell morphogenic differentiation by a caspase-dependent mechanism. Exp Cell Res 2005;307:452-464.

31. Kwan JA, Schulze CJ, Wang W, et al. Matrix metalloproteinase-2 (MMP-2) is present in the nucleus of cardiac myocytes and is capable of cleaving poly (ADP-ribose) polymerase (PARP) in vitro. FASEB J 2004;18:690-692.

32. Dawson VL, Dawson TM. Deadly conversations: nuclear-mitochondrial cross-talk. J Bioenerg Biomembr 2004;36:287-294.

33. Goto H, Nishikawa T, Sonoda K, et al. Endothelial MnSOD overexpression prevents retinal VEGF expression in diabetic mice. Biochem Biophys Res Commun 2008;15:814-820.

34. Cox MJ, Sood HS, Hunt MJ, et al. Apoptosis in the left ventricle of chronic volume overload causes endocardial endothelial dysfunction in rats. Am J Physiol Heart Circ Physiol 2002;282:H1197-H1205.

35. Lee SJ, Seo KW, Lee WS, et al. 4-Hydroxynonenal enhances MMP-2 production in vascular smooth muscle cells via mitochondrial ROS-mediated activation of the Akt/NF-kappaB signaling pathways. Free Radic Biol Med 2008;45:1487-1492. 